\title{
Projets de santé et prévention en milieu dogon du Mali .
}

Sidiki Tinta

\section{OpenEdition}

\section{Journals}

Édition électronique

URL : http://journals.openedition.org/apad/480

DOI : 10.4000/apad.480

ISSN : 1950-6929

Éditeur

LIT Verlag

\section{Édition imprimée}

Date de publication : 1 juin 1999

\section{Référence électronique}

Sidiki Tinta, « Projets de santé et prévention en milieu dogon du Mali . », Bulletin de l'APAD [En ligne], 17 | 1999, mis en ligne le 04 octobre 2006, consulté le 08 septembre 2020. URL : http://

journals.openedition.org/apad/480; DOI : https://doi.org/10.4000/apad.480

Ce document a été généré automatiquement le 8 septembre 2020

Bulletin de l'APAD 


\title{
Projets de santé et prévention en milieu dogon du Mali ${ }^{1}$.
}

\author{
Sidiki Tinta
}

1 Dans la société dogon d'aujourd'hui on rencontre deux types de prévention qui ne sont pas exclusifs l'un de l'autre. Il s'agit de la prévention liée à la survivance des pratiques traditionnelles et de la prévention de type biomédical. Dans cet article il sera essentiellement question du second type. Il est dominé par le discours de "l'éducation pour la santé".

2 L'éducation pour la santé est définie comme l'ensemble des stratégies et actions informatives développées dans le cadre de la prévention sanitaire (Bouchayer 1984 : 77). Elle se justifie essentiellement dans une logique d'efficience car il s'agit, par ce moyen, d'arriver à diminuer, sinon à annuler l'impact de certaines maladies à moindre coût. Pour cela elle mobilise des techniques comme l'information, la communication de masse, la sensibilisation, etc. Il s'agit de concevoir, sur la base d'un savoir fortement médicalisé, le message dont l'adoption peut permettre d'éradiquer les comportements nuisibles et en même temps de promouvoir ceux favorables à l'épanouissement de la santé de l'individu et du groupe. Mais le message sanitaire, parce qu'il arrive rarement à tenir compte des réalités sociales dans toute leur complexité, n'aboutit pas toujours à son objectif. Car l'individu comme le groupe social auxquels le message est destiné sont des acteurs stratégiques ayant leurs propres logiques face à des besoins à satisfaire.

En effet, ces acteurs que l'on considère comme les récepteurs de messages sanitaires, et Jean-Pierre Olivier de Sardan l'a bien souligné, n'en reçoivent pas passivement le sens. Ils le reconstruisent "...en fonction de contextes, de contraintes et de stratégies multiples. L'acteur social de base, aussi démuni ou dominé soit-il, n'est jamais un récipiendaire qui n'aurait le choix qu'entre la soumission ou la révolte" (Olivier de Sardan 1990 : 29). Il s'ensuit que deux éventualités extrêmes sont possibles. Soit le message emprunte le modèle de communication propre aux catégories biomédicales avec le risque que son contenu réel échappe au récepteur, soit il est véhiculé selon le mode de la communication locale et reste prioritairement prisonnier des catégories du récepteur. Ceci explique que certains messages connaissent plus de succès que d'autres 
et qu'un même message suscite des réactions différentes selon les individus et les groupes d'une même société. Ce sont ces situations complexes que je tenterai d'illustrer à travers quelques expériences de terrain.

\section{Situation 1}

4 Il arrive dans certains cas que le message ait été bien formulé et compris des populations. Mais du fait qu'il interfère avec d'autres informations et savoirs préalablement acquis, il est relégué au rang de savoir alternatif en attendant d'être mis à l'épreuve. Ainsi, c'est lorsque les acquis anciens échouent qu'on activera ce savoir alternatif. Il se peut aussi que le message sanitaire soit perçu comme complémentaire des acquis anciens parce que ne s'occupant que d'une dimension des besoins populaires ou des contraintes du moment. Dans tous ces cas le message finira par être intégré dans les pratiques des populations cibles. Mais cela aboutit généralement à des pratiques syncrétiques.

Expérience $1:$ Cas d'épidémie de rougeole à Sareme, dans un village du plateau, et recours à la prévention biomédicale après échec des pratiques traditionnelles.

5 Le Mali a signé la déclaration d'Alma-Ata sur les Soins de Santé Primaire (SSP) et en a fait une politique nationale à partir de 1979 (Berche, 1994 : 159). Dans le cadre de la mise en pratique de cette politique au niveau local, un certain nombre de villages ont été invités à envoyer à Bandiagara des représentants pour être formés comme agents de santé villageois (ASV). Sareme a bénéficié de la formation d'un hygiéniste-secouriste et d'une accoucheuse traditionnelle recyclée (ATR) dès 1979. Ceux-ci devaient servir de relais au niveau villageois dans l'éducation sur les problèmes de santé et les méthodes de prévention, par exemple ils devaient expliquer localement l'intérêt de la vaccination contre les maladies infectieuses et pour la prévention des épidémies. À la fin de leur formation, ces ASV réunirent les villageois pour leur faire part de ce qu'ils avaient appris. Depuis, il n'y a eu aucune application pratique des informations données jusqu'en 1992 comme le constate l'hygiéniste-secouriste lui-même :

"Lorsque les autorités sanitaires de Bandiagara demandèrent deux personnes à former, le village nous a désignés. On nous a appris comment on fait les premiers soins, le pansement, les prises de comprimés dont l'aspirine et la nivaquine et comment être propre, à quoi cela sert. On nous a appris beaucoup de méthodes pour prévenir certaines maladies comme la rougeole. À notre retour nous avons dit tout cela aux gens du village. Mais on dirait à l'époque que cela n'intéressait personne. Ils ont dit tout simplement qu'ils ont entendu et que c'est bien pour le village. Mais personne n'a fait quelque chose dans ce sens; personne aussi n'est venue nous consulter encore..." (HD, hygiéniste-secouriste).

6 Cette attitude s'explique par le fait qu'on se fiait aux pratiques préventives traditionnelles de sacrifice et de libation sur les autels et fétiches pour prévenir toutes les infortunes. Et cela aurait apparemment fonctionné jusqu'en 1992, quand une épidémie de rougeole éclata dans le village. L'échec de toutes les pratiques traditionnelles pour endiguer le mal amena les populations à consulter leurs ASV pour y parvenir.

"En 1992 il y a eu l'épidémie de rougeole. On a fait une première réunion autour de ce problème. On nous a dit qu'il fallait activer nos traditions, c'est-à-dire faire des sacrifices sur les vieux objets du village. On disait que c'est faute de les avoir négligé que l'épidémie est entrée dans le village. Nous avons sorti de l'argent dans la caisse du groupement villageois ${ }^{2}$ pour faire le nécessaire. Mais l'épidémie continuait. On a fait une deuxième réunion. C'est au cours de celle-ci que le chef de village a rappelé ce que nous leur avions dit depuis longtemps. Il a été décidé de nous confier le 
problème. On a sorti encore de l'argent dans la caisse du groupement. J'ai été voir l'infirmier de Sangha qui est venu faire la vaccination. À partir de cet instant il ya eu un mieux".

Expérience 2 : Mort d'une épileptique dans un puits à Bandiagara et recours aux pratiques traditionnelles et modernes.

On rencontre le syncrétisme dans la prévention de maladies aussi graves socialement que l'épilepsie. Traditionnellement, lorsqu'un épileptique tombait dans un puits ou une mare, cette eau n'était plus consommée. Les puits étaient généralement refermés définitivement ou en tout cas pour très longtemps, surtout lorsque le malade y mourait. Un cas de chute mortelle d'une épileptique dans un puits de Bandiagara le dix-huit février 1997 a donné lieu à des pratiques syncrétiques nouvelles. C'est dans le puits dit bundu kapi ("puits de Kapi") du nom du chef de quartier qu'une fillette de douze ans est tombée au cours d'une crise épileptique. On l'a retrouvée morte.

Juste après cet événement, conformément aux pratiques traditionnelles, le puits a été fermé. Mais en même temps on a consulté un ancien auxiliaire du service d'hygiène qui habitait le quartier. Celui-ci a dit que si on traitait le puits avec un certain nombre de produits, il n'y aurait aucun risque de contagion. Ce qui veut dire que l'auxiliaire de santé lui-même adhérait aux conceptions locales de la contagiosité de l'épilepsie ${ }^{3}$. Toujours est-il que le quartier a cotisé pour l'achat des produits dont de l'eau de Javel. Après le traitement $d u$ puits, les notables du quartier sont allés consulter des marabouts, Bandiagara étant une ville fortement islamisée. Les marabouts conseillèrent d'enlever cent fois le contenu d'une "outre à puiser" (do:yaba) du puits et de verser cette eau, ensuite de refermer le puits jusqu'au lundi suivant. Il était acquis qu'à partir $\mathrm{du}$ lundi suivant on pourrait recommencer à utiliser l'eau de ce puits. En tout la fermeture au public du puits dura à peu près une semaine.

Le lundi 24 février, le puits fut rouvert au public. Mais la plupart des gens que j'ai interrogés m'ont dit qu'ils utilisaient cette eau à d'autres fins (travail de banco, abreuvage d'animaux, linges, etc.) et non pour la consommation (boisson, cuisine, etc.). Quelques uns ont dit que, puisque le puits avait été traité, il n'y avait pas de raison de ne pas consommer son eau.

10 La ville de Bandiagara compte environ 9.485 habitants. Le système d'adduction d'eau qui date d'avant l'indépendance n'est pas fonctionnel depuis quelques années du fait que l'échelle d'entretien du château d'eau est cassée. Le système d'approvisionnement en eau se base donc essentiellement sur les puits. Or, dans toute la ville, il n'y a guère que deux ou trois puits pérennes, dont celui-là. De sorte que chaque année à partir du mois d'avril, la ville connaît de graves problèmes d'approvisionnement en eau. A titre d'exemple, en 1995 et 1996 le seau d'eau s'achetait à certains moments à Bandiagara autour de 200 francs CFA.

11 Dans un tel contexte on ne peut pas se permettre de fermer un point d'eau où tomberait un épileptique. Mais à l'opposé on ne doit pas ignorer la survivance des conceptions traditionnelles à propos de cette maladie. On adapta donc la tradition au contexte réel du moment en choisissant une voie médiane qui associe système de croyance local (fermeture du puits selon une tradition plus ancienne et consultation de marabouts suivant la tradition islamique plus récente) et savoir médical moderne (appel à l'auxiliaire de santé). On s'appuya simultanément sur l'avis du détenteur d'un savoir moderne et sur les croyances religieuses pour légitimer la réouverture du puits en contrariant quelque peu les pratiques traditionnelles. 
Expérience 3 : La maladie de l'oiseau, le PEV, les guérisseurs.

vaccination (PEV), une des composantes des SSP, et de la maladie de l'oiseau sada. Cette entité nosologique populaire, que l'on impute à un oiseau souvent mythique, est identifiée dans certains cas, en médecine moderne, à un tétanos néonatal ou à un accès pernicieux (convulsions). La santé publique a inscrit le tétanos parmi les six maladies infectieuses qui sont au cœur des objectifs du PEV et elle tente de prévenir le paludisme à travers notamment la nivaquinisation.

13 Apparemment la prévention biomédicale n'interfère ici aucunement avec le savoir populaire parce que ce qui est identifié du côté médical comme du tétanos ou une forme de paludisme apparait comme une toute autre pathologie dans les conceptions populaires, aussi bien sur le plan étiologique que sur le plan de la dénomination.

En outre, parallèlement aux vaccinations du PEV, les femmes continuent à recourir aux pratiques traditionnelles pour prévenir la maladie de l'oiseau. Ainsi, la femme enceinte doit-elle siffloter lorsqu'elle entend le cri de l'oiseau pour éviter que celui-ci "prenne" le fotus. De même, lorsque cet oiseau traverse son chemin, elle doit jeter une pierre devant elle. Elle aurait ainsi chassé le nyama de l'oiseau qui pourrait sinon attendre l'enfant pour le prendre.

15 Parfois, comme chez les Mossi (Bonnet 1990), la femme enceinte ne dormira pas dehors afin d'éviter d'être survolée par l'oiseau contaminateur. Au cas où elle est amenée à le faire on lui recommandera de déposer auprès d'elle, exactement comme chez les Mossi, une calebasse remplie d'eau afin que l'ombre de l'oiseau, qui représente son âme, soit captée par l'eau au moment où son image s'y reflète.

16 On peut aussi faire porter à l'enfant une amulette faite à partir d'un des éléments de l'oiseau (plume, serres, traces laissées sur le sol) ou de certains oiseaux carnivores tels que les vautours. On trouve là des similitudes avec les pratiques d'homéopathie symbolique relevées par Bonnet (1990) chez les Mossi : elles consistent à faire des scarifications sur le visage de l'enfant, deux traits obliques en haut des pommettes par exemple, puis à introduire dans le sang une poudre dont un élément représente l'oiseau. Parfois on prévient la maladie sada par application des logiques de l'opposition, de l'incompatibilité ou de l'antipathie. Ainsi, selon un guérisseur :

"Sato fréquente les cimetières où il épie les tombes. Quand il survole une femme enceinte et que la poussière prise au cimetière tombe sur celle-là, L'enfant à naître est dit "pris" (aije) par le sato. C'est la même chose quand il survole un nourrisson ( $i$ iru dogali) "enfant n'ayant pas laissé le sein", c'est-à-dire qui est encore au sein. Pour empêcher que les enfants ne soient attrapés par le sato, il y a un arbre sur lequel le sato ne monte jamais. Il a beaucoup de noms mais les femmes l'appellent onnu "lavement", parce qu'elles l'utilisent très souvent dans le lavement des enfants pour les protéger ou les soigner contre plusieurs maladies infantiles. On enlève les feuilles et les racines de cette plante, on prépare une décoction avec laquelle on fait des lavements aux enfants et on la leur fait boire aussi. Nous faisons aussi des "amulettes" (sebi) à partir de cette plante et on les fait porter par les nourrissons".

17 Ainsi, du fait d'une impossible articulation entre les conceptions populaires du sada et celles de la biomédecine, deux types de pratiques se superposent pour la prévention d'une pathologie apparemment identique. Car on ne voit pas quelle image de l'oiseau pourrait évoquer une quelconque idée de rouille (pour le tétanos) ou de moustique (pour le paludisme). De sorte qu'une femme qui suit la vaccination PEV et qui applique plus ou moins bien la nivaquinisation peut consulter parallèlement un guérisseur ou un 
marabout pour protéger son enfant contre sada. Ce fut le cas de NT qui habite Bandiagara et que j'ai rencontrée chez le chef de village de Doukombo que l'on considère dans la zone comme l'un des meilleurs spécialistes de sada.

"J'ai fait neuf maternités. Le premier enfant est mort de cette maladie de l'oiseau. Quand j'ai fait ma seconde maternité une vieille de Bandiagara m'a conseillée d'aller voir le chef de village de Doukombo dés que j'aurai fini avec mes quarante-cinq jours. Elle m'a demandé d'amener cinq cents francs avec moi. C'est ce que j'ai fait. Effectivement le chef m'a donné une amulette à mettre au cou de l'enfant contre paiement des cinq cents francs. Mais il m'a conseillé aussi de ne pas interrompre cette pratique. Après chaque accouchement, dès qu'un nom est attribué à l'enfant je viens chercher l'amulette. Cela ne prend pas du temps parce qu'il en a plusieurs déjà fabriquées. Tu donnes l'argent et il prend une pour te donner. Son travail m'a beaucoup réussi car depuis que j'ai commencé avec lui, tous mes enfants n'ont jamais eu cette maladie".

Le fait qu'une mère accepte de parler de la maladie de l'oiseau est déjà en soi une évolution des conceptions, car dans les villages, même de nos jours, on n'en parle pas devant les femmes encore en âge de procréer, sauf à utiliser des euphémismes du genre "c'est la chose d'en haut" (mana bagna).

19 Mais, sauf à espérer que quelques cas de sada avérés soient soignés et guéris par la médecine moderne, la rencontre entre discours médical et conceptions populaires dans ce domaine ne semble pas pour demain. Certes, les agents de santé ont parfois tenté de faire le rapprochement entre sada et paludisme par exemple par le biais d'un symptôme commun. En effet, les populations reconnaissent que le sada se manifeste essentiellement par le raidissement de l'enfant; de même qu'elles reconnaissent que certaines formes de paludisme (wobu) font raidir. Mais elles n'établissent aucun lien entre wobu et sada car pour elles le sada imputé à l'oiseau est une maladie infantile alors que wobu atteint tout le monde et est causé essentiellement par un déséquilibre alimentaire.

Situation 2

Dans certains cas, ce n'est pas le message lui-même dans sa formulation qui fait obstacle à son adoption, mais le déficit en connaissances ou la manière dont celles-ci sont transmises de l'émetteur au récepteur. Ce déficit peut être à l'origine d'un heurt inutile du message avec les conceptions locales. Ceci entraîne l'échec de l'action d'éducation sanitaire en raison d'un dysfonctionnement dans le dialogue entre l'émetteur et le récepteur. Ce type d'incompréhension se nourrit de multiples contradictions : développement observé par le récepteur différent de celui promis par l'émetteur, émetteur non congruent avec le message, exigences des populations divergentes de celle du message sanitaire, etc.

Expérience 1 : Le projet SSP à Bandiagara à travers les campagnes de vaccination et de lutte contre les maladies diarrhéiques

21 Lors de sa première phase dite de "coup de balai", qui va de 1987 à 1990, le PEY avait pour objectif de vacciner $80 \%$ des enfants de 0 à 6 ans et des femmes en âge de procréer. Cet objectif a été loin d'être atteint dans certaines zones comme à Bandiagara où le discours médical n'a pas tenu compte des effets secondaires des vaccins tels que fièvres et éruptions cutanées. C'est seulement quand les mères d'enfants vaccinés s'en plaignent qu'on leur demande de donner de l'aspirine. Cela suscite diverses interprétations de la part des populations, qui vont de la remise en cause des objectifs du PEV à celle de l'efficacité des vaccins. Ce phénomène se rencontre aussi chez 
d'autres populations voisines comme les Bambara, les Bozo et les Marka du cercle de Djenné (Tinta 1992). Voici ce qu'en dit une mère :

"On nous demande de faire vacciner nos enfants. Nous amenons des enfants sains qui reviennent malades. Quand on explique cela aux dogotoro, ils nous disent de donner de l'aspirine aux enfants. À quoi sert l'aspirine? À soigner [jogno!]. Ils rendent donc nos enfants malades pour qu'on paye leurs médicaments".

Le fait de n'avoir pas prévenu les mères sur l'éventualité d'effets secondaires et les conceptions que celles-ci ont déjà de l'aspirine sont à l'origine du malentendu. Mais celui-ci en cache un autre parfois plus important où il est fait allusion au planning familial.

"Ils nous avaient demandé d'avoir moins d'enfants. Est-ce que ce n'est pas ce qu'ils veulent faire ainsi ?"

Ce soupçon d'un objectif inavoué du PEV se complique de quelques cas extrêmes de décès d'enfants "vaccinés" contre la rougeole par exemple. Ainsi, dans un village deux femmes déclarent avoir perdu leurs enfants lors d'une épidémie de rougeole en 1995 alors qu'ils auraient été vaccinés. La question soulevée ici est celle de l'efficacité des vaccins dont on sait de source médicale que dans l'ensemble elle n'atteint jamais les $100 \%$ hormis le cas unique du vaccin contre la variole.

24 À cela s'ajoute l'inefficacité due aux conditions d'acheminement et de conservation des vaccins et aux vaccinations foraines. Certains agents du PEV nous ont parlé de la difficulté que rencontrent les équipes mobiles à assurer les rappels. Ainsi, certains enfants vaccinés lors d'un premier passage ne sont plus revus du tout ou le sont hors du délai normal de rappel. Ce qui fait que du côté médical ils sont considérés comme n'ayant pas été vaccinés alors que pour les populations ils le sont. Partant, certaines mères considèrent que la prévention biomédicale par la vaccination est inefficace et qu'au contraire elle est susceptible de nuire à la santé de leurs enfants.

Quant au discours médical sur la prévention et le contrôle des maladies diarrhéiques par les Sels de réhydratation orale il s'est souvent limité à dire aux mères "lorsque vos enfants font la diarrhée, donnez leur le SRO". Or, le SRO n'est pas un médicament en soi contre la diarrhée. Il sert uniquement à prévenir la déshydratation chez l'enfant diarrhéique. Cette précision ayant manqué au discours médical, les populations ont compris que le SRO était un traitement anti-diarrhéique. Après avoir essayé ce produit, exceptionnellement donné mais généralement vendu alors qu'officiellement il devrait être gratuit, les mères commencèrent à se plaindre :

"Ce n'est pas un bon médicament. J'en ai donné trois à mon enfant, son ventre continue à couler. On nous trompe toujours. D'ailleurs on avait dit que c'était gratuit. Seules les premières femmes qui sont allées au dispensaire l'ont eu sans payer. Nous autres on a acheté".

On a alors tenté de réagir par des explications qui ne trouvent pas de référent dans les conceptions populaires. Dans ce discours de rattrapage, la réhydratation est traduite par "compensation de la perte d'eau du corps" gesu le ni go ma ka kano. Or, dans les conceptions locales, si la diarrhée est considérée comme une maladie qui se traite, elle n'implique pas une perte d'eau du corps chez le malade...

Ce même déficit dans la manière dont le savoir médical est transmis a été observé à Koro avec le programme de lutte contre le ver de Guinée. À l'époque, le service d'hygiène du cercle qui était chargé de la campagne de sensibilisation a fait dire aux populations que l'eau de pluie était responsable du ver de Guinée et que pour s'en 
prémunir il fallait boire l'eau de puits. Durant l'hivernage qui a suivi les populations consommèrent l'eau des puits mais ne furent pas protégées contre le ver de Guinée. Il s'est produit même une épidémie de ver de Guinée, car les puits n'étaient pas protégés par des margelles hautes. En cas de pluie ils étaient immergés sous l'eau. Or, les conditions favorables à l'infection sont réunies dès lors que l'eau de boisson est tirée de points d'eau de surface tels que des mares, des puits à marches, ou des citernes (Muller, 1979).

"On nous a dit de boire l'eau de puits pour éviter le ver de Guinée. Nous l'avons fait et pourtant on dirait que le ver n'attendait que cela pour attraper tout le monde. Nous ne comprenons rien de nos dogotoro actuels"

Expérience 2 : Le projet "schisto" à Bandiagara qui pensent que c'est surtout une maladie de l'enfance, pas assez grave pour mériter un traitement. Elle est censée guérir d'elle-même lorsque la personne affectée grandira. Dans cette conception la maladie est liée à l'eau. Certains ajoutent même que c'est lorsqu'on nage dans l'eau que de petites bêtes rougeâtres pénètrent dans le corps en passant par l'anus. Quelques rares informateurs disent que cette maladie est due au fait de consommer beaucoup de "pain de singe". Cette forme de bilharziose urinaire est dite mogo banu, c'est-à-dire le "mogo rouge" à cause de l'aspect sanguinolent de l'urine du malade. Cependant, lorsque la maladie continue alors que la personne a atteint une vingtaine d'années, cela est considéré comme un phénomène anormal. On recherchera alors une bonne médication surtout par crainte que le "mogo rouge" n'évolue vers une autre forme plus grave, le "mogo blanc" (mogo pilu) qui évoque les symptômes de la gonococcie. considéré comme une maladie mais comme le signe de la fertilité d'un enfant. La conception dogolu de la bilharziose établit même qu'un jeune garçon qui n'est pas "affecté" a de fortes chances d'être stérile. On l'appelle généralement les "menstrues des hommes" [anam puno). On le distingue cependant d'une forme de "mogo blanc" qu'on assimile à une maladie sexuelle. Certains l'identifient sous le nom "d'urine blanche" [isari pilu) à cause des pertes blanches qu'elle provoque chez le malade.

Tout comme les Dogon les Bambara de l'office du Niger ${ }^{4}$ distinguent deux formes de pathologies assimilées à la bilharziose. La première forme, qu'ils nomment grosin, est 
jugée normale parce qu'étant le signe de la virginité chez les enfants. Elle n'implique généralement pas la recherche d'une action préventive ou curative. La deuxième forme est appelée damajalan, "besoin sec", et est censée n'atteindre que les personnes adultes. Elle est socialement jugée grave parce qu'elle connote un comportement frivole dans un couple. C'est le signe qu'un homme ou une femme ont été coupables d'infidélité. Ce qui est" honteux".

C'est dans ce contexte d'une double conception de la bilharziose que le "projet schisto" devait inscrire son action préventive. Dans les zones où l'on percevait la bilharziose comme un mal bénin il y a eu très peu de résistance face au discours préventif. Au contraire, le fait même que les conceptions locales considéraient déjà que la bilharziose était transmissible par contagion simplement en enjambant l'urine du malade facilitait l'adoption des mesures préventives. Mais en zone dogolu en particulier, où le programme s'est largement investi, on a considéré que les informations contenues dans les messages sanitaires étaient une agression à l'encontre du savoir local.

"Dans nos traditions on n'a jamais appris que le mogo est une maladie. Nos parents avaient même peur que leur enfant ne connaisse pas le mogo; personne n'a jamais soigné le mogo. Après sa période, il disparaît comme les règles de femme après une semaine. Aujourd'hui les dogotoro disent que c'est une maladie donnée par les eaux de mare. On nous demande de ne pas consommer ces eaux et de ne pas laisser les enfants entrer là-dedans. En tout cas, tant que nous ne verrons pas de nos propres yeux, notre "pensée" est ailleurs..."

Ici, le message de prévention, bâti sur les conceptions biomédicales de la bilharziose qui privilégient l'aspect parasitologique de la maladie, heurte des conceptions locales qui n'attribuent pas le statut de malade à l'individu "affecté" de la bilharziose. Ce décalage explique l'insuccès de ce projet dans certaines zones. Mais pour des raisons difficilement compréhensibles ce projet continue à survivre de ses échecs sans changement des stratégies d'intervention.

Expérience 3 : Le planning familial dans le projet SSP Bandiagara

Parfois, le discours préventif connaît des échecs parce que le message n'est pas congruent avec l'émetteur. L'un des exemples concerne le projet Soins de Santé Primaires (SSP) de Bandiagara qui avait pour objectif entre autres de promouvoir la santé maternelle et infantile à travers des thèmes comme les méthodes contraceptives, l'espacement des naissances ou leur limitation.

Pour animer la première phase de ce projet qu'on a appelée "phase de sensibilisation", on a recruté dans la capitale régionale une jeune sage-femme, célibataire sans enfant, pour la raison qu'il s'agissait d'un problème de femmes... J'ai rencontré par hasard cette jeune femme à Kendié, un des chefs-lieux d'arrondissement du cercle, où il existe un dispensaire et une maternité.

37 Cette sage femme avait deux handicaps majeurs à gérer pour que son message passe. Le premier, c'était son statut de femme célibataire n'ayant jamais enfanté, donc ne pouvant disposer d'aucune expérience dans ce domaine pour en parler. Lors de la réunion d'information, en présence de l'infirmier local qui servait de traducteur, après que la jeune sage-femme finit d'exposer ses idées, une femme qui avait environ la quarantaine demanda la parole. Dans ses propos il y avait trois questions essentielles adressées à la sage-femme. Premièrement, on lui demandait si elle était mariée ; la réponse fut négative. Deuxièmement, on lui demandait si elle avait des enfants; la réponse fut négative. Troisièmement, on voulait savoir si elle en avait déjà eu et s'ils étaient morts ; la réponse fut encore négative. C'est alors que l'intervenante dit : "Je ne 
sais pas ce que tu vas nous apprendre, ma fille; il vaut mieux qu'on ne continue pas cette réunion". Les femmes se levèrent et se dispersèrent.

Le deuxième handicap concernait son hôte, l'infirmier en poste à Kendié, polygame et père de plus d'une dizaine d'enfants. C'est après cette réunion que va se jouer une scène qui a permis d'établir le lien entre d'une part la réaction des femmes et d'autre part le statut de la sage-femme, son lieu de d'hébergement et le fait de s'être fait accompagner par l'infirmier lors de la réunion. Car, après la fin prématurée de la réunion, une des femmes, parente à plaisanterie de l'infirmier, s'est rendue au domicile de celui-ci pour parler à ses femmes en présence de la sage femme.

"Vous et vos maris êtes tous des égoïstes. Il y a combien d'hommes dans ce village qui ont autant de femmes que lui? Il y a combien de femmes qui ont autant d'enfants que vous? Et vous demandez aux autres d'arrêter ou d'avoir peu d'enfants. Et notre sœur qui est venue de loin, qu'est-ce que vous lui avez dit?..."

Il s'agissait de dire que ce ne sont pas cet infirmier et cette sage-femme qui sont les personnes les mieux indiquées pour tenir un tel discours.

Dans une autre localité du cercle, à Ningari, les femmes ont eu une réaction plus souple. Lorsqu'on leur parla de planning familial, elles répondirent "qu'elles sont des queues et non des têtes" et que pour parler de cela il fallait qu'elles en parlent d'abord avec leur mari. Mais les femmes, en rapportant la parole de la sage-femme à leurs maris, ont mis plutôt l'accent sur l'aspect de la limitation des naissances. Dans cette localité la secte musulmane Wahabia est dominante. C'est un de ses responsables qui est aussi le président de l'Association Malienne pour l'Unité et le Progrès de l'Islam qui va répondre au nom des hommes :

"C'est Dieu qui donne les enfants. Ceux qui essayent d'en limiter le nombre commettent une faute grave envers la loi divine. Nous sommes ici fidèles à cette loi, donc nous sommes contre cette parole des dogotoro".

Lorsque je travaillais sur le système de santé du cercle de Djenné (Tinta, 1992), l'AMUPI de cette localité s'était opposée à toute idée de planning dans les mêmes termes. Cette incursion des hommes dans ce qui a été faussement considéré comme le domaine des femmes montre bien le déficit du discours médical. Les hommes se considérant traditionnellement comme les maîtres de toute décision engageant la famille, le fait d'avoir touché en premier lieu les femmes a pu être vécu comme une atteinte à leur autorité. Pour se venger de ce sentiment de frustration, les hommes n'auraient-ils pas avancé l'argument religieux?

Expérience 4 : Les soins de santé maternelle et infantile à Bandiagara

Un volet non moins important du projet SSP concernait les consultations pré et postnatales. Un des objectifs assignés aux matrones des dispensaires était de détecter les grossesses à risque pour les référer au plus tôt à l'instance sanitaire supérieure. Mais ces matrones pratiquent souvent des actes dont elles ne maîtrisent pas le contenu et qui choquent inutilement les femmes qui viennent consulter. Il s'agit principalement $\mathrm{du}$ toucher vaginal. Très souvent, quand on demande aux matrones ce qu'elles font lorsque les femmes enceintes arrivent, elles citent le toucher vaginal, puis l'analyse des urines et la pesée. Les femmes sont très réticentes au toucher vaginal parce que, disent-elles, "nous n'acceptons pas qu'on s'amuse avec notre corps".

Ainsi à Djenné (Tinta 1992), comme dans certaines maternités de Bandiagara, les matrones elles-mêmes reconnaissent que celles à qui l'on a fait un toucher vaginal lors de la première consultation ne reviennent plus. Or les matrones ne sont pas 
compétentes pour le pratiquer en raison de leur faible niveau de connaissance. Ce geste qui n'est pas nécessaire à leur niveau a un impact négatif sur la fréquentation des maternités.

Le problème posé ici est double: d'une part celui du niveau réel des connaissances médicales des agents (qu'on peut appeler aussi celui d'un déficit réel en connaissances), et d'autre part celui de la nécessité de certains actes susceptibles de heurter les traditions et conceptions populaires dans tout ce qui a un rapport avec l'éthique du corps. En l'occurrence le toucher vaginal donne l'occasion aux matrones de valoriser leur savoir faire qui est très souvent banalisé par les femmes. En effet, pour celles-ci la consultation prénatale (ou postnatale) se résume à la pesée de la mère et de l'enfant et s'appelle, en milieu dogon mais aussi de façon générale en Afrique de l'Ouest, "pesée" : peze en dogon, pesigol en peul, peseli en bambara, etc. Les matrones pour prouver qu'elles font mieux qu'une simple pesée tenteront ce "geste médical" qu'est le toucher vaginal.

\section{Situation 3}

L'objectif "la santé à moindre coût" peut ne pas être atteint parce que les populations cibles peuvent s'inscrire dans des logiques de "détournement" ou "d'accaparement" (Olivier de Sardan 1990). Selon cet auteur, le principe de détournement signifie que les populations utilisent les opportunités fournies par un programme pour les mettre au service de leurs objectifs alors que dans la logique de l'accaparement ce sont des groupes particuliers qui s'approprient les projets pour acquérir du prestige ou l'accroître. Les responsables d'un projet de santé tendent à le présenter en bloc afin qu'il soit accepté en bloc par les villageois et en face les populations procèdent à une mise en scène pour faire croire qu'elles acceptent l'offre afin de bénéficier des avantages matériels liés à ce projet (Tinta, 1999).

Expérience 1 : Le programme "paludisme" à Bandiagara

Le programme paludisme est une composante des SSP. Il figure dans les activités "Prévention et contrôle des endémies locales" (Berche 1994: 203). Dans le cadre de ce programme, après une formation d'environ deux à quatre jours, un certain nombre de guérisseurs ont été associés non seulement en raison de leur influence sur les populations, mais aussi de leur proximité. Le séminaire de formation a été assuré par les agents du Centre de santé du cercle et ceux du Centre régional de médecine traditionnelle (CRMT). Ce dernier collabore avec les guérisseurs organisés en Association de thérapeutes traditionnels (ATT). La qualité de membre de ces associations donne à son titulaire le droit d'avoir une carte et parfois une blouse pour les collaborateurs les plus fidèles. Après cette formation, ces guérisseurs savent répéter intégralement le message qui leur a été enseigné. Ils savent dire, comme cette vieille guérisseuse, que le moustique donne le paludisme :

"Ce qui donne précisément le paludisme ce sont les moustiques. La manière dont les moustiques transmettent le paludisme! Le paludisme s'il vient, souvent ça commence par une personne. Le moustique après avoir bu le sang du malade, s'il arrive à piquer celui qui n'a pas le paludisme, ça le contamine, dans ma "pensée" $[\ldots] "$

47 Ils savent dire qu'avec la nivaquine on peut guérir le paludisme ou le prévenir (inde aganaw gia kauji) "l'empêcher d'attraper une personne"

"[...] Pour ne pas être atteint de paludisme, on peut prendre trois nivaquines par semaine. Mais une fois qu'on est atteint, pour soigner l'enfant jusqu'à onze mois c'est un comprimé par jour; soit un demi comprimé le matin et un demi le soir 
pendant trois jours. De douze mois à 5 ans, tu prends un comprimé le matin et un le soir pendant trois jours. De six ans à dix ans, c'est un comprimé le matin, un à midi et un le soir, pendant trois jours. De onze ans à quinze ans, il faut donner deux comprimés le matin et deux le soir pendant trois jours. À seize ans et plus, on donne deux comprimés le matin, deux à midi et deux le soir pendant trois jours".

Mais ce discours relève plus d'une logique dans laquelle s'inscrit l'élève averti par son maître de l'éventualité d'un contrôle inopiné sur le savoir reçu que d'une réelle appropriation du savoir transmis. En effet, au moment de la formation les guérisseurs ont été prévenus que des superviseurs passeraient vérifier qu'ils ont bien compris et retenu la formation. Pour prouver donc qu'ils ont été de bons élèves, ils répéteront à tout venant les leçons apprises. Mais dès l'instant qu'ils comprennent que l'interlocuteur n'a rien à voir avec les formateurs, les discours changent, du moins ce fut le cas en ce qui me concerne. Car cette vieille, comme d'autres guérisseurs formés, une fois que je leur avais que je n'avais rien à voir avec le programme paludisme et que mon intérêt portait plus sur ce qu'ils savaient avant que sur ce qu'ils savaient après cette formation, ont produit des discours presque inverses au fil des entretiens. Je me suis rendu progressivement compte que pour ces guérisseurs, en tout cas pour la plupart, l'information donnée par les agents de santé à propos du paludisme était du simple "vernis" en dessous duquel persistait un savoir autre, à partir duquel ils ajustaient leurs pratiques. Ainsi, le sujet devenait impersonnel ("on"). Désormais le discours tenu n'était plus "le moustique donne le paludisme. La nivaquine soigne le paludisme" mais "on nous a dit que le moustique donne le paludisme et que la nivaquine protège ou soigne contre le paludisme".

"Ce que nous savions avant dans la tradition, certains disent que le wobu, nous on dit argu en jamsay, est donné par la saleté logo notamment les aliments "sales" ou "avariés" (nyowon). Parfois c'est le "changement d'eau" (ni duluwu) qui amène le argu; d'autres disent que c'est Dieu ou le "mauvais vent" (onyo monyu); mais en milieu dogon partout il est reconnu que le argu vient avec l'hivernage. Il existe en d'autres saisons, mais c'est avec la verdure de l'hivernage qu'on voit beaucoup le argu. Selon les Dogons, c'est la période où il pleut beaucoup, on mange des fruits sucrés comme le yulo (parkia biglobosa), le mignu (Butyrospernum parkii), le kambu (Sabasenegalens-sglabiflora). C'est ce qui donne le argu".

50 Ce n'est plus le moustique mais un déséquilibre alimentaire, avec un excès de sucré, qui donne le paludisme. Et la thérapie curative comme préventive consiste dans l'application de la logique de l'opposition pour rétablir cet équilibre.

"Depuis nos ancêtres nous utilisons les plantes pour prévenir le wobu. Nous utilisons les plantes amères en décoction pour boire ou se laver et en "fumigation" (uguru), surtout à l'approche de l'hivernage".

51 Certaines des recettes médicinales contre le wohu sont de l'ordre du secret professionnel. Mais il y en a une qui a été fréquemment citée et qui m'était connue. D'ailleurs, elle est répandue dans presque toutes les régions dogon. À son propos on peut parler d'un savoir commun partagé. Il s'agit d'une décoction à partir de jeunes bourgeons de guru (Guiera senegalensis) ajoutée à du tamarin (fruits ou feuilles) et du piment. Cette préparation repose toute la nuit. Le lendemain matin, elle est filtrée et prise à jeun par toute la famille. C'est la méthode classique de prévention contre le wohu. L'association de l'amer, le guru, de l'acide, le tamarin, et de l'épicé, vise à la fois à protéger et à guérir. Si le guru par son amertume sert à prévenir le wohu en éliminant 
l'excès de sucré dans le corps, le tamarin sert en plus à purger, et le piment à "tuer" comme on le dit le kefi ${ }^{5}$ ou "petit du kefam".

52 Certains guérisseurs écartent ainsi la causahté moustique dans leur discours.

"Nous soignons beaucoup de cas de wobu mais si ce n'est pas les dogotoro, nous on ne sait pas ce qui donne cette maladie. Nous savons seulement que c'est plus fréquent pendant l'hivernage" .

D'ailleurs, si les conceptions populaires et les conceptions biomédicales convergent pour appeler le paludisme wohu ou argu, c'est au niveau de la détermination des symptômes que le consensus est le plus manifeste. En effet, l'élément central des conceptions locales c'est "faire de la température" (godu numo),"corps échauffé". Mais les guérisseurs ont fait remarquer au cours de leur formation qu'on pouvait faire de la température pour plusieurs raisons. Ils ont cependant distingué ces différents cas d'une catégorie particulière qui s'accompagne d'autres symptômes tels que maux de tête, vomissement (moussant ou jaune), diarrhée, "courbature" (godu yimu), manque d'appétit et parfois "raidissement" (magne). C'est donc la fièvre qui accompagne ces symptômes et que les Dogon appellent wohu, argu ou kejam que les guérisseurs et leurs formateurs ont convenu de retenir comme correspondant au paludisme.

S'il y a cette convergence minimale entre les agents de santé moderne et les guérisseurs, c'est parce que ces derniers sont dans une logique de "détournement" et "d'accaparement". Jusque dans un passé relativement récent, les guérisseurs ne jouissaient que d'une légitimité traditionnelle tirée des coutumes et d'une légitimité charismatique. En cela, la médecine dite traditionnelle dont ils étaient censés être les animateurs apparaissait comme une médecine parallèle ou non officielle par opposition à une médecine officielle bénéficiant d'une légitimité légale-rationnelle, d'où son nom de " médecine conventionnelle".

L'absence de légitimité légale a longtemps entrainé la mise en cause des guérisseurs dans l'exercice de leur fonction. Ainsi, tout guérisseur pouvait être, en cas d'accident survenu à son patient, poursuivi et sanctionné, alors que son homologue de la médecine moderne bénéficiait d'une certaine immunité (Tinta 1993: 221). En conséquence, pour les guérisseurs, l'adhésion à ce programme, qui signifiait une collaboration avec la médecine officielle, pouvait les gratifier d'une légitimité légale protectrice. Pour être formé dans le cadre du programme, le candidat devait être membre d'une Association de thérapeutes traditionnels. Or, l'une des raisons de l'adhésion des guérisseurs à ces ATT, c'est justement l'acquisition de la carte de membre, perçue comme un certificat de légalisation de la profession de guérisseur.

Ce phénomène n'est d'ailleurs pas nouveau. Il date de l'époque où, à la suite de la déclaration d'Alma Ata (1978), l'Organisation mondiale de la santé voyait dans la collaboration entre les médecines traditionnelle et moderne le seul moyen d'étendre une couverture sanitaire accessible à tous. On parlait alors de la revalorisation de la médecine traditionnelle.

Le Mali avait déjà anticipé sur cette politique de l'OMS. en créant dès 1975 une Association des thérapeutes traditionnels. En 1982 l'Institut national de recherche sur la pharmacopée et la médecine traditionnelles (INRPMT) est fondé qui deviendra plus tard la Division Médecine Traditionnelle (DMT) de l'Institut national de recherche en santé publique (INRSP). 

compétence, notamment à partir de critères comme la reconnaissance de la légitimité de leur pratique par la communauté dont ils étaient ressortissants. À l'issue de cette évaluation, une carte était remise au candidat. Il y avait quatre cartes différentes qui étaient distribuées: la carte de membre de l'ATT, celle d'herboriste, la carte professionnelle des thérapeutes traditionnels et l'attestation de collaboration avec la DMT (ancien INRPMT). Mais on ne savait jamais quels étaient les critères d'attribution des différentes cartes et les bénéficiaires eux-mêmes ne voyaient pas de différence parce qu'à leurs yeux elles avaient toutes valeur de carte professionnelle. De plus, un certain nombre de guérisseurs ont profité de cette carte pour augmenter abusivement leurs tarifs, ou faire valoir leur droit à être considéré comme agent de santé, (Pairault 1993 : 324). Pour ces raisons, les autorités maliennes mirent fin à cette expérience dès 1983, soit une année après sa naissance. Mais elle allait renaître sous une autre forme à partir de 1986 avec la multiplication des ATT et leur relative autonomie par rapport à la structure nationale, la DMT. Désormais l'adhérent n'a droit qu'à une carte de membre d'une ATT. Mais pour les guérisseurs cette nouvelle carte de membre de l'ATT est perçue comme ayant la même valeur que l'ancienne carte professionnelle. L'adhésion aux ATT et aux programmes de développement sanitaire comme celui sur le paludisme se situe donc toujours pour eux dans une logique de conquête et de renforcement de la légitimité légale qui leur manque.

le cadre du programme paludisme il était prévu que les agents de santé fassent des supervisions périodiques des activités des guérisseurs formés. Le passage des agents de supervision a été lui-même perçu par ceux envers qui s'exerçait ce contrôle comme légitimant. Lorsque le superviseur, représentant de l'autorité légale, choisit dans un village d'aller chez tel guérisseur plutôt que chez tel autre cela peut être interprété comme une manière de le valoriser.

Enfin, l'adhésion au programme devenait une source de revenu supplémentaire, donc un enjeu de pouvoir. Le comprimé de nivaquine qui était vendu cinq francs aux guérisseurs formés était revendu par eux dix francs auprès de la population.

61 Cette stratégie de quête d'une rétribution à la fois légale (acquisition d'une carte de membre d'une ATT), symbolique (visite des superviseurs) et économique (vente de médicaments) du fait d'une collaboration avec la médecine moderne était encore perceptible lors du colloque Médecine traditionnelle et sida qui s'est tenu les 7 et 8 février 1994 à Bamako. Ce colloque, qui regroupait dans une relative parité médecins et guérisseurs, a permis à ceux-ci de jouer ouvertement leur stratégie. Ils ont lu notamment des motions demandant la mise en place de conditions juridiques d'égalité entre médecine traditionnelle et médecine moderne, la formation des guérisseurs à travers des séminaires, mais aussi une rétribution financière compensatoire de leur engagement dans la lutte contre le sida et d'autres maladies. Mais vite les bons élèves d'hier ne respectent pas la leçon, au nom d'une certaine éthique sociale.

"Au moment de la formation on nous a dit de ne pas vendre de comprimés à celui qui ne peut pas acheter tout un traitement. Nous avons accepté mais c'est difficile quand c'est une connaissance qui vient vous le demander".

Cette déviation des guérisseurs a débouché parfois sur des situations conflictuelles. Ce fut le cas pendant l'hivernage 1994. Cette année-là, le Département d'épidémiologie et d'affections parasitologiques (DEAP) de Bamako, qui participe au programme national de lutte contre le paludisme, avait envoyé une équipe sur place, dont des 
entomologistes. Ceux-ci sont passés dans certaines concessions pour capturer des anophèles. Mais la nivaquinisation ayant été mal faite, il y eut une épidémie de paludisme à Bandiagara. La prise en sous-dose de nivaquine annulait tout bénéfice de protection et toute possibilité de diagnostic par les examens biologiques, notamment par la goutte épaisse. Face à l'ampleur du phénomène les populations ont soupçonné l'équipe du DEAP d'avoir volontairement semé l'épidémie.

De son côté, l'équipe médicale, pour essayer de mieux diagnostiquer les cas de paludisme, demanda l'arrêt des pratiques prophylactiques par la nivaquine. Ceci a été interprété par les populations en général, y compris les guérisseurs, comme une défaillance du savoir médical. En effet la nivaquine, à cause de son amertume -d'ailleurs on l'appelle communément le "médicament amer" [Itoi jeru] -, était perçue comme efficace contre le wobu et non contre le paludisme imputé aux moustiques. Ainsi, telle jeune femme venue acheter deux comprimés de nivaquine chez le guérisseur du coin m'a dit :

"Je viens d'un mariage où j'ai mangé beaucoup "d'aliments doux" (do : nyala elelu). Depuis mon arrivée je ne me sens pas bien. On dirait qu'on a frappé tout mon corps. J'ai peur que ce soit le argu; je vais boire ces médicaments pour diminuer la douceur dans mon sang".

Une autre raison explique la pratique du sous-dosage par les guérisseurs et les populations. Dans la nivaquinisation, l'accent avait été particulièrement mis en direction des femmes enceintes. Or, dans les conceptions locales, la nivaquine prise à certaines doses devient un abortif. Finalement, outre la nivaquinisation, on a proposé aux populations l'usage de "moustiquaires et de rideaux trempés", de fumigènes, etc. Mais ces moyens semblent à la fois manquer d'efficacité et d'efficience. On sait que le Mali figure parmi les pays les plus pauvres du monde. Pour un paysan c'est déjà un luxe que d'avoir une moustiquaire ou un rideau, à plus forte raison d'en avoir pour toute sa famille et de trouver les moyens de les imprégner chaque année. Ensuite, les spécialistes ont indiqué que le pic des moments de piqûre des anophèles correspond à l'aurore et à l'aube, les seuls moments où l'on a toutes les chances de ne pas trouver le paysan sous une moustiquaire : s'il ne revient pas des champs, il y va. Enfin, nombre de personnes sont, ou se disent, allergiques à la nivaquine : or le quinimax en comprimé est trop rare et trop coûteux pour constituer un produit alternatif. Sa boîte d'environ trente comprimés coûte environ 3.000 FCFA.

Expérience $2:$ Le programme national d'éradication du ver de Guinée à Karakindé, un village du cercle de Koro

Il y a des moments où le message sanitaire, bien qu'il transgresse les conceptions locales de la maladie et de sa prévention, donne l'illusion d'avoir été accepté et intégré en raison des stratégies de détournement et d'accaparement que développent les acteurs sociaux. Ce n'est que lorsque ceux-ci auront atteint leurs objectifs réels, ou auront réalisé qu'ils sont inaccessibles, qu'apparaîtront des stratégies inverses de rejet. C'est le cas du Programme national d'éradication du ver de Guinée (PNEVG), qui formait des Agents de santé villageois (ASV) pour des activités d'hygiène et d'assainissement (éducation pour la santé, protection des points d'eau, promotion des latrines etc.) et la surveillance épidémiologique du ver de Guinée (Ndiaye et al. 1995 : 14). Dans la région de Mopti, la Direction nationale de l'Hydraulique et de l'énergie (DNHE) en collaboration avec l'UNICEF a réalisé et équipé des points d'eau salubres dans les cercles de Douentza, Koro et Mopti en 1994. 
Dans le cadre de la coopération bilatérale entre le Mali et le Japon, un projet d'hydraulique villageoise visant l'éradication du ver de Guinée a été financé et exécuté. Il a particulièrement couvert le cercle de Bandiagara où il a eu quelques succès car l'équipe mobile de sensibilisation comptait en son sein des agents de santé bien équipés. Ainsi, avec les microscopes, elle a essayé de montrer la différence entre l'eau des sources protégées, comme celle des puits aménagés dans le cadre du programme, et l'eau de surfaces non protégées, comme les mares ou les étangs.

Cette expérience corrobore les conceptions locales qui voient la prévention comme l'accomplissement d'un certain nombre d'actes concrets. C'est pourquoi elle a immédiatement suscité quelques réactions d'adhésion au moins dans les discours : dans l'un des villages où j'ai assisté à cette démonstration visuelle, les villageois avaient promis de ne plus utiliser l'eau des mares pour leur consommation. Mais il est difficile de prévoir comment ce discours de quelques individus ayant assisté à l'expérience peut se traduire dans les pratiques du groupe villageois.

D'autre part, il y a des zones où le message a été à l'origine de conflits locaux. Ainsi, Koro est un village d'environ 1.000 habitants, où les populations s'approvisionnaient en eau dans les puits pendant la saison sèche et dans les mares pendant l'hivernage. Il y avait trois puits à buses auxquels se sont ajoutés deux puits-pompes acquis dans le cadre du PNEVG. Le village a bénéficié de la formation d'un ancien hygiéniste-secouriste comme agent du PNEVG. Une équipe de supervision devait périodiquement passer pour faire le point des activités.

Malgré ces efforts, le PNEVG à Karakindé, de l'avis général des acteurs, fut un échec, au moins jusqu'en 1997, période de la fin de mes enquêtes dans ce village. Pour comprendre les raisons de cet échec, il faut rappeler le contexte, les acteurs et les conflits locaux à propos de ce programme.

L'infection par le ver de Guinée est une maladie parasitaire des zones où les populations tirent leur eau potable d'étangs et de puits peuplés de cyclopidés infectés (Muller 1979). Par contre, l'infection ne se manifeste pas avec des sources d'eau courante ni à des puits ayant une circonférence de moins de trois mètres (Muller 1979). La dracunculose est l'une des maladies les plus faciles à contrôler voire à éradiquer, si l'on considère que: a) la période d'infectivité est limitée à quelques semaines, b) l'infection humaine doit impérativement se produire chaque année, c) il n'y a pas de réservoir animal important, d) la transmission est limitée à de petits foyers facilement délimités. Une fois que la transmission est interrompue pendant une saison, l'infection disparaît à moins qu'elle ne soit réintroduite de l'extérieur. Les moyens les plus sûrs et efficaces de contrôler la transmission et de bloquer le cycle de réinfection sont : a) le tamisage de l'eau de boisson avec un linge, b) le traitement chimique des points d'eau avec du Temephos, c) l'amélioration des sources d'eau potable.

1 Des méthodes biologiques, comme l'introduction de petits poissons, ont été préconisées (Muller 1971). Autre méthode, la distribution de tamis-filtres, qui aurait permis d'éradiquer le ver de Guinée dans trois villages de la région de Banfora, au Burkina Faso, deux ans après la mise en place des activités (Gbary et al. 1987).

Mais les moyens préventifs qui réussissent ailleurs ont rencontré une forte résistance de la part des populations de Karakindé. Dans ce village il y a apparemment deux conceptions différentes sur l'étiologie et la prévention du ver de Guinée. Il y a une conception que partagent les membres d'un lignage du clan damna. Pour ceux-ci, 
l'origine du ver de Guinée se trouverait dans la transgression d'un "interdit" (donwon) lié au hérisson.

"Les gens de Jemne [du nom du quartier qu'ils habitent] ont pour patronyme Goro. Ils sont originaires $d u$ domno. Ils ont été tous "pris pour être amenés" 6 ici. Le hérisson est leur interdit. Ils sont venus avec et ils ne l'ont pas oublié. Ils ne doivent pas le toucher ou le manger, même pas le voir. Si c'est le cas, il faut lui offrir tout ce qu'on a en sa possession. Quand un Goro transgresse cet interdit, une épidémie de ver de Guinée se déclare dans le quartier. Souvent même sans faire exprès un Goro qui passe sur les traces du hérisson, même s'il ne fait pas de ver de Guinée, il aura des démangeaisons au corps".

Dans ce lignage on pense que la seule manière de se protéger contre le ver de Guinée, c'est de respecter l'interdit du hérisson, ou, en cas de violation de cette règle, de procéder à des rites de purification collectifs.

"II faut que tous les descendants Goro du quartier subissent un rituel de purification sinon l'épidémie se poursuivra. Une femme qui se marie dans ce quartier, à moins que cela ne soit aussi l'interdit de son lignage paternel, n'est pas concernée par cette "règle d'évitement" (ta). À partir du moment où elle entre dans leur famille et mange leur repas, même si elle avait l'habitude d'attraper le ver de Guinée, elle sera immunisée tant qu'elle y reste. Mais à supposer qu'elle touche un hérisson ou le mange quelque part, après qu'elle rentre au domicile conjugal et si elle se sert du même pot ou boit dans la même jarre que les autres membres de la famille, ceux-ci attraperont le ver de Guinée. Si leur fille se marie avec toi, que vous ayez un enfant, tant que le hérisson n'est pas votre propre interdit, l'enfant est aussi épargné. Il se limite à la mère. Au cas où l'enfant le touche il faut purifier son corps avant qu'il ne s'approche de sa mère. Autrement, si l'enfant est toujours au sein et s'il tête ainsi sa mère, ils partageront les effets" (AG).

Mais il y a aussi ceux qui pensent que le ver de Guinée est lié à l'eau, notamment l'eau des mares dans laquelle aurait pénétré une personne déjà atteinte, même s'ils déclarent parfois ignorer ce qui donne le ver de Guinée. Pour ceux-ci, la prévention passe par la surveillance des points d'eau pour que les malades n'y pénètrent pas. En outre certains guérisseurs connaîtraient des médicaments préventifs. Ainsi, l'agent du PNEVG qui lui-même fut un ancien malade du ver de Guinée disait :

"Avant cette formation, ce qu'on savait faire pour ne pas contracter le ver de Guinée, c'est aller voir les guérisseurs. Il y a certains qui donnent des médicaments préventifs. Parfois cela peut marcher, mais parfois non. Il y a aussi ceux qui donnent des médicaments pour qu'au cas où tu attrapes la maladie, la partie ne s'enfle pas. Ainsi, il y aura moins de douleur" (DG, di, H44, ASV).

Un guérisseur du village m'a dit que sa recette était faite à partir de deux plantes: konloro (non identifié) et ouron bauri (Xirneninarnericana). Mais la réussite de cette prévention a pour condition que le bénéficiaire s'interdise de manger les feuilles ou les fruits de ces plantes. Autrement, du fait cette transgression même, il attrapera le ver de Guinée.

Dans la prévention traditionnelle il est fait grand usage de cette logique de l'incompatibilité entre ce qui a servi à fabriquer le charme protecteur et un autre usage ultérieur par le bénéficiaire.

On voit déjà que si cette seconde conception du ver de Guinée n'a rien de contradictoire avec la conception biomédicale, elle apparaît comme difficilement conciliable avec la première. Et pourtant tout semblait avoir bien commencé pour les tenants des deux conceptions lorsque l'agent, du retour de sa formation, avait tenu une réunion d'information. 
"[...] C'est à Madougou que j'ai été formé. À mon retour, j'ai réuni les villageois pour les informer de ce que j'ai appris à propos du ver de Guinée : comment maintenir le village propre, surtout les abords des puits pour que les eaux usées ne se déversent pas dedans ; comment détecter les cas de ver de Guinée et comment appliquer des produits antiseptiques et des bandages occlusifs sur la plaie jusqu'à la sortie totale du ver ; comment on peut éviter la maladie en filtrant toutes les eaux avant leur consommation. Ils ont été informés à cette occasion que des tamis-filtres allaient bientôt arriver à raison de 150F CFA l'unité et que le village allait bénéficier du forage de deux puits-pompes. Donc tout le monde était d'accord pour participer à l'action. Le lendemain, des réunions ont été organisées par les hommes de chaque quartier pour informer et impliquer les femmes. Tout cela s'est bien passé".

Le premier lot de tamis a été distribué gratuitement dans toutes les familles. Un superviseur m'a même dit qu'à Karakindé toutes les femmes utilisaient déjà les tamis pour filtrer l'eau de consommation, car, lors de la supervision, il avait observé que toutes les femmes qui partaient ou qui revenaient du puits tenaient leur tamis en main. Puis l'agent du PNEVG a constaté un relâchement. L'explication des femmes était que les premiers tamis arrivés étaient usés. Un second lot arriva. Bien que ce deuxième lot ait été vendu et non gratuit, la population en acheta massivement. Ce qui suscita chez l'agent l'espoir d'un succès total du programme :

"[...] Au début, j'avais peur. Je m'étais dit que c'est parce que les tamis étaient gratuits que les gens l'ont accepté. Mais avec le second lot où, dans certaines familles, on en a pris jusqu'à dix, j'ai cru maintenant que mon projet va réussir. Mais avant l'hivernage beaucoup avait abandonné le travail, à commencer par les gens de Jemme".

79 Le second relâchement correspondait à peu près à la période où les deux puits-pompes avaient été réalisés. En ce sens, tout le village semble avoir joué une stratégie de groupe d'acceptation des tamis afin de pouvoir bénéficier de ces forages pour résoudre le problème de la disponibilité et de la qualité de l'eau. Car, auparavant, les trois puits existants atteignaient des profondeurs de 50 mètres. Les eaux tirées de ces puits essentiellement à la main avaient un goût "amer" (jeru) et ne pouvaient servir à laver les linges blancs parce que ne moussant pas au savon ordinaire.

80 À cause de la mauvaise qualité de cette eau, qu'il fallait en plus se procurer dans des conditions pénibles, certains enseignants mutés dans ce village préféraient parfois démissionner. Le jour de marché hebdomadaire, les ressortissants des villages environnants apportaient avec eux leur eau de boisson. En outre, les femmes du village étaient obligées d'aller laver leur linge à plus de sept kilomètres dans les villages voisins.

81 C'est pourquoi l'annonce de l'obtention de nouveaux forages, liés à l'acceptation du PNEVG, avait été accueillie avec soulagement. Mais une fois les puits acquis, la stratégie de groupe s'est effacée face au respect de la tradition. Pour les gens de Jemne, le seul moyen d'éviter le ver de Guinée restait soit de respecter l'interdit soit de se soumettre à un rite de purification après l'avoir violé. En recourant à d'autres moyens de prévention comme filtrer l'eau, on admettait avoir violé l'interdit, tout en refusant la réparation rituelle.

De plus, pour l'ensemble du village, on se rend compte que le tamis a joué un rôle folklorique dans la stratégie de détournement des objectifs du PNEVG. Selon mes propres observations et les informations qui m'ont été données sur place, la plupart des tamis amenés par les femmes sur les points d'eau n'ont jamais servi à filtrer l'eau. Le geste qui consistait à les apporter au puits et à les rapporter à la maison servait aux 
femmes à prouver à leurs maris que l'information donnée avait été entendue et acceptée. On se servait des tamis pour d'autres activités ménagères (tamiser la farine de mil, filtrer le jus de tamarin, etc.) que filtrer l'eau. Il y avait là une logique économique. Un tamis de ce genre coûtait sur le marché autour de 500 FCFA alors que dans le cadre du programme il était vendu à 150 FCFA.

Une autre raison de la démobilisation a été que l'eau des puits-pompes s'est révélée de la même qualité que celle des autres puits, alors qu'on espérait que ce serait de l'eau douce, et qu'elle n'aurait pas le goût saumâtre de l'eau du village. Si les forages nouveaux ont soulagé les populations du problème de la disponibilité de l'eau à moindre effort (désormais on utilise les pompes manuelles à la place des cordes tirées à la main), ils n'ont pas résolu celui de sa qualité. Quand les premières pluies ont commencé à tomber, les gens se sont rués sur les eaux de mares plus faciles d'accès et au meilleur goût, sans utiliser les tamis.

84 L'argument le plus souvent avancé par la population pour justifier ce non-usage des tamis restaient qu'ils étaient abîmés. Mais certaines femmes reconnaissaient que le filtrage de l'eau constituait une "gêne" (ganu) à leurs activités principales, la cuisine et la culture. Pendant l'hivernage où tout le monde est pressé par les activités agricoles, on n'a pas de temps à perdre pour filtrer l'eau. Dans leur pensée il n'y avait pas de lien entre filtrer l'eau des mares et éviter le ver de Guinée.

Le PNEVG a pourtant fait un effort pour placer à côté des principales mares des tamis dont tout le monde pouvait se servir, mais sans succès :

" [...] Quand les femmes ont commencé à dire que les tamis sont vieux ou usés, j'en ai parlé aux superviseurs. On m'a envoyé quelques tamis que j'ai placé à proximité des principales mares car rares sont ceux qui allaient désormais au puits. Il n'y avait que deux ou trois familles qui utilisaient la pompe en plus des deux familles d'enseignants. Mais à ma grande surprise les tamis commençaient à disparaître alors que ceux qui étaient restés n'étaient jamais utilisés puisqu'ils n'étaient jamais mouillés. C'est la preuve qu'on utilisait les tamis à d'autres fins" :

D'autres familles avaient émigré dans des hameaux de culture où elles résidaient pendant toute la durée de l'hivernage. Ainsi dispersées et loin du regard de l'agent et des superviseurs du PNEVG mais aussi des puits du village (certains hameaux se situent au-delà des $5 \mathrm{~km}$ du village), la mare devenait leur seule source d'approvisionnement en eau.

De toutes les façons la prévention était devenue presque inutile car les premiers cas de ver de Guinée étaient déjà apparus. Ce qui a fait dire aux populations que l'idée même de tamis-filtres serait à l'origine de l'épidémie de ver de Guinée, car selon elles, auparavant, il n'y avait jamais eu dans leur village autant de cas de ver de Guinée.

Au mois d'août, les superviseurs, furieux de l'ampleur de l'épidémie dans ce village, ont réuni quelques notables pour leur faire des remontrances. Apparemment cela a ouvert la voie à un conflit entre le PNEVG et les villageois. Ce conflit va se répercuter par ricochet sur l'agent local, lui-même natif du village.

"[...] Quand les superviseurs sont arrivés, j'étais absent. J'avais déménagé dans mon hameau où je cultivais. Il paraît qu'ils ont sévèrement critiqué les villageois en disant que sur toute l'étendue de la République ils sont les seuls à avoir autant humilié le PNEVG par leur non respect des consignes données. Après leur départ, les villageois s'en prirent à moi. Pour eux c'est moi qui ai envoyé un rapport aux superviseurs pour les informer de la situation. C'est un peu vrai aussi parce que cela faisait partie de mon rôle. Mais ce qu'ils n'ont pas pu dire aux superviseurs, c'est à 
moi qu'ils l'ont dit. Le fait que ce sont les tamis qui ont amené le ver de Guinée, avant c'était surtout de la rumeur. On ne me l'avait jamais dit directement. Mais depuis le passage des superviseurs les gens venaient directement me le dire. Ils ont dit aussi que désormais il faut arrêter les traitements que je faisais aux malades. Les attaques les plus virulentes venaient de mes anciens camarades de classe, c'est ce qui m'a fait le plus mal". comprendre et à adhérer au PNEVG que le reste de la population analphabète. Mais ce sont ceux-là mêmes qui se montraient les plus incompréhensifs. On serait tenté d'expliquer cette réaction des anciens scolarisés (que les villageois appellent lecole perdi, les perdus de l'école) vis-à-vis de l'agent comme la manifestation d'une compétition pour la conquête de nouvelles positions sociales statutaires. En effet, après les échecs scolaires qu'ils avaient connus ensemble, l'agent et ses anciens camarades occupaient en apparence la même position sociale d'élèves recalés, de "perdus de l'école". Mais l'agent, de par sa nouvelle fonction, était en voie d'ascension sociale et occupait une position statutaire plus valorisante. Ses anciens camarades s'employaient par rivalité à hypothéquer ce succès en se mettant à l'avant-garde de la désapprobation populaire.

du PNEVG n'a pas trait seulement aux stratégies des uns et des autres. Il y a aussi un facteur méconnu, négligé, ou non pris en compte par les acteurs du PNEVG. Les auteurs sont à peu près d'accord pour dire qu'en Afrique la transmission survient principalement à la saison des pluies (Petit et al., 1988). Or, en période hivernale, ce village d'environ 1.000 habitants, compte moins de la moitié de sa population habituelle. Les autres vont résider dans les hameaux, loin des points d'eau protégés. Il est vrai qu'à cette saison parcourir des kilomètres à la recherche d'une source d'eau protégée constitue une véritable gêne pour les activités agricoles. Ils ont donc recours aux sources potentiellement contaminées.

Conclusion

93 L'éducation pour la santé est donc une opération complexe de négociation entre éducateurs et bénéficiaires dont personne ne peut présager l'issue, qui dépend des contextes et des contraintes qui entourent le groupe cible. Dans tous les cas, la réussite ou l'échec d'une action en éducation pour la santé n'est pas prédéterminée uniquement à partir d'un problème de formulation et de communication de bons messages. Son impact est aussi fonction de la prise en compte de l'ensemble des déterminants sociaux et psychologiques des conduites pouvant influencer sur l'ordre social en général et la santé en particulier. Car, comme l'a souligné Yannick Jaffré, "en face de l'émetteur se 
trouve un récepteur pris dans les réseaux de son désir, de son environnement objectif et des règles du jeu social de son milieu" (1991: 59-60).

La modernité sanitaire dogon dans le domaine de la prévention est devenue un véritable marché au sens d'un espace de propositions d'offres et de demandes émanant d'acteurs stratégiques multiples. Les bénéficiaires accepteront les offres parfois par nécessité, souvent par conviction, et aussi par stratégie de captation des avantages liés à l'offre. Dans le meilleur des cas on évolue dans une logique syncrétique : les pratiques traditionnelles se superposent à celles de la modernité. Dans d'autres cas, les pratiques traditionnelles paraissent incompatibles avec les propositions modernes, soit parce que la modernité est perçue comme une transgression de la tradition, soit parce que l'efficacité de la modernité est jugée assez convaincante pour suppléer à la tradition. Entre ces deux pôles se cachent des conflits d'acteurs qui expliquent et justifient l'échec ou la réussite des projets de développement sanitaire.

\section{BIBLIOGRAPHIE}

Anonyme 1995. Rapport sur les stratégies additionnelles du programme national d'éradication du ver de Guinée. Bamako.

Berche, T. 1990. "Anthropologie et santé publique. Une approche du paludisme au Burkina Faso". In Fassin et Jaffré (eds), Sociétés. Développement et Santé. Paris : Karthala

Berche, T. 1998. Anthropologie et santé publique en pays dogon. Paris : Karthala.

Bouchayer, F. 1984. "Les logiques sociales des actions en éducation pour la santé". Sciences Sociales et Santé II (3-4).

Bourdarias, F \& Le Palec, A. 1995. "Guérisseurs et médecins, interactions et stratégies, à propos du colloque Médecine traditionnelle et SIDA". Journal des Anthropologues 60.

Delamare, J. (ed.) 1992. Dictionnaire des termes de médecine. Paris : Éditions Maloine.

Gbary, A.R., Guigemde, T.R, Ouedraogo, J.B.,1987. "La dracunculose, un fléau éradiqué dans trois villages du Burkina Faso par l'éducation sanitaire" Bulletin de la Société de Pathologie Exotique $80: 30-395$

Hopkins, D.R. 1987. "Dracunculiasis eradication ; a mid-decade status report" American Journal of Tropical Medicine and Hygiène 37(1) : 115- 118.

Jaffré, Y. 1991." Anthropologie de la santé et éducation pour la santé" Cahiers Santé 1 : 406-14

Le Marcis, F. 1998." Quelques maladies contagieuses dans le Jallubeeri (Mali)", Bulletin $d u$ Programme de recherche VII :" Interactions entre les systèmes de santé publique et les conceptions et pratiques populaires relatives à la maladie (Afrique de l'Ouest)".

Muller, R. 1971." Dracunculus and dracunculiasis". Advances in Parasitology 9 : 73-151.

Muller, R. 1979."Guinea worm disease : epidemiology, control and treatment". Bulletin of the World Health Organization 57 (5) : 683-689. 
Ndiaye, M., Peries, H., Maiga, A. 1995. Opportunités d'intégration des programmes d'éradication du ver de Guinée dans la santé à base communautaire, Statut, Draft 1.

Olivier de Sardan, J.P. 1990. " Sociétés et développement. In Fassin \& Jaffré (eds) : Sociétés. développement et santé. Paris : Ellipses.

Olivier de Sardan, J.P 1995. Anthropologie et développement, Paris :Karthala.

Pairault, C. 1993. "À propos d'efficacité médicale en Afrique Noire" In Brunet-Jailly (ed). Se soigner au Mali. Paris, :Karthala-ORSTOM.

Pene. P., Andre, L.J., Rougemont, A., Bourgeade, A., Barbe, P. 1980. Santé et médecine en Afrique tropicale. tome 2, Paris : Doin Editeur.

Tinta, S \& Pairault, C. 1990. Enquête socio-sanitaire dans le cercle de Bandiagara (1989-1990), Bamako : INRSP. multigr.

Tinta, S. 1991. Le financement des coûts récurrents de la santé dans le cercle de Bandiagara au Mali. Vol.II : Acceptabilité et participation. Bamako : INRSP, multigr.

Tinta, S. 1993. Créer un centre de santé communautaire? Le cas de Kamba (arrondissement de Sangha). Bamako : INRSP. multigr.

\section{NOTES}

1.Cet article est extrait d'un chapitre de ma thèse portant sur "Les conceptions autour de la transmission de la maladie et les pratiques préventives chez les Dogon du Mali" (Tinta 1999)

2.Nouvelle forme d'association villageoise mixte d'entraide et de solidarité. Ces groupements tirent leurs ressources de travaux collectifs effectués par les membres au profit d'un particulier qui les paye ou de dons venant d'autres associations et organismes. Ces ressources sont utilisées à des fins festives ou pour le financement d'actions d'intérêt général.

3.Rappelons que si l'épilepsie n'est pas contagieuse, elle est considérée comme l'étant par nombre de populations africaines.

4.Je tiens cette information d'une communication personnelle de mon ami Samba Diop qui a travaillé à l'office sur les maladies liées à l'eau.

5.Ce terme emprunté au peul désigne le germe du kejam qui dans cette langue serait synonyme du wobu dogon; d'ailleurs, dans beaucoup de dialectes dogon on utilise parfois le terme peul de kefam pour parler de paludisme à la place du terme dogon. 6.Ainsi appelle-t-on ceux qui ont été razziés et réduits à l'esclavage par les Peul : ajeru de $a=$ prendre + jer $u=$ amener . 


\section{AUTEUR}

\section{SIDIKI TINTA}

INRSP, BP Ini. Bamako, Mali 